\title{
Reprodutibilidade do exame de medida da camada de fibras nervosas da retina por meio da polarimetria de varredura a laser
}

\author{
Reproducibility of retinal nerve fiber layer measurement by scanning laser \\ polarimetry
}

Fernanda Bon Duarte ${ }^{(1)}$

Augusto Paranhos Jr. ${ }^{(2)}$

Roberta Andrade Galhardo ${ }^{(3)}$

Luciano Ribeiro Arabe Abdanur ${ }^{(4)}$

João A. Prata Jr. ${ }^{(5)}$

Paulo Augusto de Arruda Mello ${ }^{(6)}$

\section{RESUMO}

Objetivo: Analisar a reprodutibilidade das medidas da espessura da camada de fibras nervosas da retina intra e inter-observador.

Material e Métodos: As medidas da camada de fibras nervosas foram realizadas em 26 olhos normais por três examinadores diferentes, os quais tomaram três imagens de cada olho. Com base nestas imagens foi executada a média das imagens pelo programa do aparelho. A cada examinador foi solicitado que escolhesse a melhor imagem para cada exame. Foram analisados os resultados obtidos com a melhor imagem, bem como os da média das imagens. Foi calculada a média da espessura da camada de fibras nervosas nas regiões superior, nasal, temporal e inferior e suas relações. Determinou-se a reprodutibilidade nestas circunstâncias tanto inter como intra-observador por meio de teste de análise de variância por postos de Friedman.

Resultados: Na análise intra-observador houve ótima reprodutibilidade do exame. Para o examinador G (glaucomatólogo) os valores de $p$ foram os seguintes: região superior $=0,48$; inferior $=0,89$; temporal $=0,30$ e nasal $=0,89$. Para o examinador $R$ (residente) os valores de $p$ foram os seguintes: região superior $=\mathbf{0 , 8 9}$; inferior $=$ 0,24 ; temporal $=0,89$ e nasal $=0,30$ e para o examinador $\mathrm{T}$ (tecnóloga) os valores de $\mathbf{p}$ foram os seguintes: região superior $=\mathbf{0 , 7 6}$; inferior $=$ 0,70 ; temporal $=0,22$ e nasal $=0,63$. Na análise inter-observador, não foi observada diferença estatisticamente significante das variáveis isoladas considerando-se a média das imagens ( $p$ superior $=0,52$; inferior $=0,37$; temporal $=0,43$ e nasal $=0,52$ ). Considerando-se as relações entre as regiões temos que apenas a relação $\mathrm{S} / \mathrm{I}$ mostrou diferença estatisticamente significante. As demais relações: $S / T, S / N$, I/T, I/N e T/N não mostraram diferença estatisticamente significante. Para a melhor imagem observou-se diferença estatisticamente significante entre os três examinadores para as variáveis superior/temporal $(p=0,02), n f e r i o r / t e m p o r a l ~(p=0,03)$ e temporal $/$ nasal $(p=0,007)$ e superior/inferior $(p=0,037)$ e para as médias de espessura nos quadrantes inferior $(p=0,04)$ e temporal $(p=0,003)$.

Conclusões: A análise dos dados indica ótima reprodutibilidade do exame intra-observador, e na avaliação inter-observador os melhores resultados de reprodutibilidade são obtidos quando a média das imagens fornecida pelo programa do equipamento é empregada.

Palavras-chave: GDx; Analisador de fibras nervosas da retina; Camada de fibras nervosas da retina. 


\section{INTRODUÇÃO}

Glaucoma é uma neuropatia que ocasiona perda de células nervosas da retina de maneira irreversível. Por conseguinte, o diagnóstico e tratamento precoce são muito importantes ${ }^{1}$. Classicamente, o exame de campo visual tem sido empregado com a finalidade de detectar tal perda celular, entretanto, estudos sugerem que ocorre considerável dano glaucomatoso antes de que possam ser evidenciadas alterações de campo visual ${ }^{1,2}$. Estudos histológicos realizados em humanos que recentemente tinham se submetido a exame de campo visual por perimetria automática, revelam uma perda importante de células ganglionares sem que pudesse ser evidenciada uma redução da sensibilidade na perimetria estática ${ }^{3}$.

Portanto, atualmente têm-se buscado métodos propedêuticos mais sensíveis e, dentre eles, destaca-se o exame de camadas de fibras nervosas da retina. Sabe-se que a perda das fibras nervosas da retina é a alteração inicial da lesão glaucomatosa ${ }^{2}$.

Embora a camada de fibras nervosas possa ser avaliada in vivo usando a oftalmoscopia com filtro "red-free" e a fotografia, essas técnicas não permitem uma avaliação quantitativa da espessura da camada de fibras nervosas ${ }^{4,5}$. A polarimetria de varredura a laser (PVL) é uma nova técnica para uma análise quantitativa da espessura da camada de fibras nervosas da retina ${ }^{6,7}$. Apresenta uma boa sensibilidade $(96 \%)$ podendo ser o primeiro teste para rastreamento do glaucoma ${ }^{8}$. Entretanto, outros autores encontraram uma menor sensibilidade $(87,3 \%)$ e recomendam a associação com os dados clínicos e semiológicos do paciente ${ }^{9}$.

Weinreb e cols ${ }^{7}$ estabeleceram uma excelente correlação entre a espessura da camada de fibras nervosas da retina obtida com a polarimetria de varredura a laser e os achados histo-patológicos em retinas de macacos: aproximadamente 1 grau de retardamento corresponde a 7,4 $\mu \mathrm{m}$ de espessura.

A polarimetria de varredura a laser consiste em um feixe de laser infra-vermelho de diodo polarizado $(780 \mathrm{~nm})$ que atravessa os meios oculares em direção à retina. Como a camada de fibras nervosas se comporta como um meio birrefringente, ocorre mudança do estado de polarização da luz. Presume-se que esta propriedade se deva aos microtúbulos das células ganglionares da retina. A unidade de captação da luz polarizada quantifica esta mudança da polarização em graus que é definida como retardamento, e correlaciona com a espessura da camada de fibras nervosas da retina. Uma varredura completa consiste em 65536 pontos retinianos individualizados ( $256 \times 256$ pixels).

O objetivo deste estudo é analisar a reprodutibilidade das medidas da camada de fibras nervosas da retina intra e interobservador pela técnica de polarimetria de varredura a laser em pacientes normais.

\section{MATERIAL E MÉTODOS}

Foram realizadas medidas da espessura da CFN da retina com o analisador de fibras nervosas $\mathrm{GD}_{\mathrm{X}}{ }^{\mathrm{MR}}$ (Laser Diagnostic
Technologies, Inc. San Diego, CA), por três examinadores diferentes (1 oftalmologista com especialização em glaucoma-G, 1 residente - R e 1 tecnólogo-T) em indivíduos voluntários normais no Setor de Glaucoma do Departamento de Oftalmologia da Universidade Federal de São Paulo- Escola Paulista de Medicina.

Cada examinador obteve três imagens de boa qualidade de ambos os olhos dos voluntários, segundo os critérios de centralização. Utilizando essas três imagens foi efetuada a sua média (parte do programa do aparelho), sobre a qual foi feita demarcação do limite da papila e cálculo da espessura da CFN da retina. Após isso, cada observador escolheu a melhor imagem, sobre a qual foi demarcado o limite do disco óptico com a elipse regulável do programa do aparelho e realizado o cálculo da espessura da CFN da retina.

As medidas foram sempre feitas com as pupilas não dilatadas e com a luz da sala sempre acesa. Foram consideradas para os cálculos as médias de todas as medidas da espessura da CFN em cada um dos quatro quadrantes seguindo a seguinte orientação topográfica: $120^{\circ}$ para superior; $120^{\circ}$ para inferior, $70^{\circ}$ para nasal e $50^{\circ}$ para temporal.

Tomou-se para efeitos de cálculos a média das medidas da espessura da camada de fibras nervosas dos 1500 pontos mais espessos das regiões superior e inferior e dos 1500 pontos medianos das regiões nasal e temporal, conforme utilizado pelo programa do equipamento para cálculo dos índices.

Os parâmetros acima e as relações entre eles (fornecidas pelo equipamento): superior/inferior (S/I), superior/temporal $(\mathrm{S} / \mathrm{T})$, superior/nasal $(\mathrm{S} / \mathrm{N})$, inferior/temporal $(\mathrm{I} / \mathrm{T})$, inferior/ nasal $(\mathrm{I} / \mathrm{N})$ e temporal $/$ nasal $(\mathrm{T} / \mathrm{N})$ foram analisados para a melhor imagem e a média das imagens.

Pelo teste de variância por postos de Friedman. foi avaliada a reprodutibilidade inter e intra-observador tomando-se em conta a média das imagens e a melhor imagem escolhida por cada examinador. Para as comparações múltiplas foi utilizado o método de Dunn. Foi adotado valor de $\alpha=5 \%$

\section{RESULTADOS}

A amostra constou de 13 pacientes ( 26 olhos ) sendo 06 do sexo feminino e 07 do sexo masculino, a idade variou de 22 a 49 anos (média: 28,6 anos $\pm 6,43$; mediana: 28 anos).

No que se refere à média das imagens, não houve diferença estatisticamente significante entre as medidas feitas pelos 3 examinadores em nenhum dos parâmetros analisados: superior $-\mathrm{p}=0,52$; inferior $-\mathrm{p}=0,37$; nasal $-\mathrm{p}=0,52$; temporal $p=0,43$ (tabela 1 ). No que diz respeito às relações, apenas a relação $\mathrm{S} / \mathrm{I}$ mostrou diferença estatisticamente significante $(\mathrm{p}=0,037)$. As outras relações $(\mathrm{S} / \mathrm{T}-\mathrm{p}=0,60 ; \mathrm{S} / \mathrm{N}-\mathrm{p}=0,12$; $\mathrm{I} / \mathrm{T}-\mathrm{p}=0,76 ; \mathrm{I} / \mathrm{N}-\mathrm{p}=0,75 ; \mathrm{T} / \mathrm{N}-\mathrm{p}=0,14)$ não mostraram diferença estatisticamente significante (tabela 2).

Em relação à melhor imagem escolhida por cada examinador não houve diferença nos parâmetros superior $(\mathrm{p}=0,45) \mathrm{e}$ nasal $(\mathrm{p}=0,30)$ (tabela 1$)$, e nas relações $\mathrm{S} / \mathrm{N}(\mathrm{p}=0,29)$ e I/N 


\begin{tabular}{|c|c|c|c|c|c|c|c|}
\hline Variável & $\mathbf{N}$ & $\operatorname{Max}$ & Min & Mediana & Comparações & Diferença & $\mathbf{P}$ \\
\hline S G MI & 26 & 149,8 & 79,6 & 109,8 & $T \times G$ & Não & \\
\hline S R MI & 26 & 150,7 & 77,2 & 113,1 & $T \times R$ & Não & \\
\hline S T MI & 26 & 151,6 & 75,1 & 110,1 & $R \times G$ & Não & 0,4555 \\
\hline I G MI & 26 & 137,9 & 66,5 & 104,6 & $T \times G$ & Sim & \\
\hline I R MI & 26 & 148,2 & 65,8 & 104,0 & $T \times R$ & Sim & \\
\hline I T MI & 26 & 150,8 & 67,7 & 108,1 & $R \times G$ & Não & $0,0402^{*}$ \\
\hline T G MI & 26 & 90,2 & 31,9 & 48,5 & $\mathrm{~T} \times \mathrm{G}$ & Sim & \\
\hline T R Ml & 26 & 96,4 & 31,5 & 55,9 & $T \times R$ & Não & \\
\hline T T MI & 26 & 150,1 & 33,8 & 55,0 & $R \times G$ & Não & $0,0029^{*}$ \\
\hline N G MI & 26 & 87,2 & 35,1 & 49,8 & $T \times G$ & Não & \\
\hline N R MI & 26 & 76,7 & 34,4 & 49,6 & $T \times R$ & Não & \\
\hline N T MI & 26 & 77,5 & 37,2 & 54,6 & $R \times G$ & Não & 0,3054 \\
\hline S G M & 26 & 151,7 & 83,1 & 108,8 & $\mathrm{~T} \times \mathrm{G}$ & Não & \\
\hline SR M & 26 & 148,2 & 75,9 & 106,6 & $T \times R$ & Não & \\
\hline STM & 26 & 148,5 & 71,2 & 108,6 & $R \times G$ & Não & 0,5218 \\
\hline I G M & 26 & 145,3 & 68,1 & 101,4 & $T \times G$ & Não & \\
\hline I R M & 26 & 144,2 & 60,8 & 102,2 & $T \times R$ & Não & \\
\hline I T M & 26 & 143,0 & 67,5 & 108,0 & $R \times G$ & Não & 0,3752 \\
\hline T G M & 26 & 94,5 & 31,1 & 48,2 & $T \times G$ & Não & \\
\hline T R M & 26 & 94,7 & 31,0 & 52,4 & $T \times R$ & Não & \\
\hline $\mathrm{TTM}$ & 26 & 132,6 & 31,4 & 51,0 & $R \times G$ & Não & 0,4389 \\
\hline NG M & 26 & 81,6 & 32,5 & 49,2 & $T \times G$ & Não & \\
\hline NRM & 26 & 79,3 & 35,8 & 48,6 & $T \times R$ & Não & \\
\hline N T M & 26 & 74,0 & 35,4 & 55,1 & $R \times G$ & Não & 0,5223 \\
\hline
\end{tabular}

$(p=0,40)$ (tabela 2). Entretanto houve diferença estatisticamente significante nas relações $\mathrm{S} / \mathrm{T}(\mathrm{p}=0,02), \mathrm{I} / \mathrm{T}(\mathrm{p}=0,03)$ e T/N $(p=0,007)$ e $\mathrm{S} / \mathrm{I}(\mathrm{p}=0,039)$ (tabela 2$)$ e nos parâmetros isolados inferior $(\mathrm{p}=0,04)$ e temporal $(\mathrm{p}=0,003)$ (tabela 1$)$.

Ao avaliar a melhor imagem, aplicou-se o método de Dunn para comparações múltiplas e isolamento do observador que diferiu dos outros. Verificou-se que em relação aos parâmetros analisados isoladamente o examinador $\mathrm{R}$ diferiu dos demais quanto à espessura na região temporal e o examinador $\mathrm{T}$ diferiu dos demais quanto à espessura na região inferior. Quanto às relações observou-se que o examinador $\mathrm{R}$ diferiu dos demais na relação I/T e T/N e o observador $\mathrm{G}$ diferiu dos demais no parâmetro $\mathrm{S} / \mathrm{T}$.

A análise intra-observador, mostrou boa reprodutibilidade em todos os parâmetros analisados não havendo diferença estatisticamente significante em nenhuma medida. Considerando as três medidas obtidas pelo examinador $\mathrm{G}$ em cada região, temos que o valor de $\mathrm{p}$ para as regiões $\mathrm{S}=0,48 ; \mathrm{I}=0,89 ; \mathrm{T}=0,30$ e $\mathrm{N}=0,89$. As medidas obtidas pelo examinador $\mathrm{R}$ mostram os seguintes valores de p para as regiões: $\mathrm{S}=0,89 ; \mathrm{I}=0,24 ; \mathrm{T}=0,89$ e $\mathrm{N}=0,30$ e as medidas obtidas pelo examinador $\mathrm{T}$ têm como valores de $\mathrm{p}$ os seguintes: $\mathrm{S}=0,76 ; \mathrm{I}=0,70 ; \mathrm{T}=0,22$ e $\mathrm{N}=0,63$. Estes dados estatísticos se encontram na tabela 3 .

\section{DISCUSSÃO}

Weinreb e cols ${ }^{10} \mathrm{e}$ Chi e cols ${ }^{11}$ encontraram boa reprodutibilidade usando o analisador de fibras nervosas I (NFA I) em indivíduos saudáveis e com glaucoma. Zangwill e cols ${ }^{12}$ realizaram em 5 pacientes um estudo para avaliar a influência de três diferentes operadores sobre a reprodutibilidade da polarimetria a laser com o NFA II (novo software que possui a capacidade de exclusão dos vasos sanguíneos para determinar a espessura da CFN da retina) e concluiu que o aparelho confere medidas com boa reprodutibilidade entre os diferentes observadores em diferentes dias. Tjon-Fo-Sang e cols ${ }^{13}$ referem boa reprodutibilidade entre as relações $\mathrm{S} / \mathrm{N}$, $\mathrm{I} / \mathrm{N}$ e $\mathrm{T} / \mathrm{N}$. Os resultados obtidos com a média das imagens corroboram com os determinados por Holló e cols ${ }^{14}$ que mostram boa reprodutibilidade do exame apesar da existência de opacificações subepiteliais corneanas.

Outros pesquisadores, no entanto, relataram uma reprodutibilidade inter-observador relativamente baixa usando o NFA I ${ }^{15}$. 


\begin{tabular}{|c|c|c|c|c|c|c|c|}
\hline Variável & $\mathbf{n}$ & Max & Min & Mediana & Comparações & Diferença & p \\
\hline $\begin{array}{l}\mathrm{S} / \mathrm{I} \mathrm{G} \mathrm{MI} \\
\mathrm{S} / \mathrm{I} \mathrm{R} \mathrm{Ml} \\
\mathrm{S} / \mathrm{I} \mathrm{T} \mathrm{Ml}\end{array}$ & $\begin{array}{l}26 \\
26 \\
26\end{array}$ & $\begin{array}{l}1,33 \\
1,29 \\
1,41\end{array}$ & $\begin{array}{l}0,711 \\
0,840 \\
0,710\end{array}$ & $\begin{array}{l}1,068 \\
1,066 \\
1,007\end{array}$ & $\begin{array}{l}R \times T \\
R \times G \\
G \times T\end{array}$ & $\begin{array}{l}\text { Sim } \\
\text { Não } \\
\text { Sim }\end{array}$ & $0,0394^{*}$ \\
\hline $\begin{array}{l}\mathrm{S} / \mathrm{T} \mathrm{G} \mathrm{Ml} \\
\mathrm{S} / \mathrm{T} \mathrm{R} \mathrm{MI} \\
\mathrm{S} / \mathrm{T} \mathrm{T} \mathrm{MI}\end{array}$ & $\begin{array}{l}26 \\
26 \\
26\end{array}$ & $\begin{array}{l}3,85 \\
3,96 \\
3,58\end{array}$ & $\begin{array}{l}1,419 \\
1,222 \\
1,336\end{array}$ & $\begin{array}{l}2,422 \\
2,281 \\
2,362\end{array}$ & $\begin{array}{l}G \times R \\
G \times T \\
T \times R\end{array}$ & $\begin{array}{l}\text { Sim } \\
\text { Sim } \\
\text { Não }\end{array}$ & $0,0174^{*}$ \\
\hline $\begin{array}{l}\mathrm{I} / \mathrm{T} \mathrm{G} M \mathrm{MI} \\
\mathrm{I} / \mathrm{T} \mathrm{R} \mathrm{MI} \\
\mathrm{I} / \mathrm{T} \mathrm{T} \mathrm{MI}\end{array}$ & $\begin{array}{l}26 \\
26 \\
26\end{array}$ & $\begin{array}{l}3,58 \\
3,73 \\
3,48\end{array}$ & $\begin{array}{l}1,328 \\
1,324 \\
1,541\end{array}$ & $\begin{array}{l}2,414 \\
2,160 \\
2,351\end{array}$ & $\begin{array}{l}T \times R \\
T \times G \\
G \times R\end{array}$ & $\begin{array}{l}\text { Sim } \\
\text { Não } \\
\text { Sim }\end{array}$ & $0,0284^{\star}$ \\
\hline $\begin{array}{l}\text { S/N G Ml } \\
\text { S/N R Ml } \\
\text { S/N T Ml }\end{array}$ & $\begin{array}{l}26 \\
26 \\
26\end{array}$ & $\begin{array}{l}2,81 \\
2,93 \\
2,56\end{array}$ & $\begin{array}{l}1,375 \\
1,371 \\
1,196\end{array}$ & $\begin{array}{l}2,210 \\
2,106 \\
1,973\end{array}$ & $\begin{array}{l}T \times R \\
T \times G \\
G \times R\end{array}$ & $\begin{array}{l}\text { Não } \\
\text { Não } \\
\text { Não }\end{array}$ & 0,2914 \\
\hline $\begin{array}{l}\mathrm{I} / \mathrm{N} \text { G MI } \\
\mathrm{l} / \mathrm{N} \mathrm{R} \mathrm{Ml} \\
\mathrm{I} / \mathrm{N} T \mathrm{MI}\end{array}$ & $\begin{array}{l}26 \\
26 \\
26\end{array}$ & $\begin{array}{l}2,78 \\
2,64 \\
2,82\end{array}$ & $\begin{array}{l}1,182 \\
1,169 \\
1,078\end{array}$ & $\begin{array}{l}2,012 \\
2,019 \\
1,980\end{array}$ & $\begin{array}{l}T \times R \\
T \times G \\
G \times R\end{array}$ & $\begin{array}{l}\text { Não } \\
\text { Não } \\
\text { Não }\end{array}$ & 0,405 \\
\hline $\begin{array}{l}\text { T/N G Ml } \\
\text { T/N R Ml } \\
\text { T/N T Ml }\end{array}$ & $\begin{array}{l}26 \\
26 \\
26\end{array}$ & $\begin{array}{l}2,57 \\
2,52 \\
2,13\end{array}$ & $\begin{array}{l}0,560 \\
0,552 \\
0,542\end{array}$ & $\begin{array}{l}0,994 \\
0,974 \\
0,925\end{array}$ & $\begin{array}{l}R \times T \\
R \times G \\
G \times T\end{array}$ & $\begin{array}{l}\text { Sim } \\
\text { Sim } \\
\text { Não }\end{array}$ & $0,0073^{*}$ \\
\hline $\begin{array}{l}S / I G M \\
S / I R M \\
S / I T M\end{array}$ & $\begin{array}{l}26 \\
26 \\
26\end{array}$ & $\begin{array}{l}1,36 \\
1,32 \\
1,48\end{array}$ & $\begin{array}{l}0,749 \\
0,729 \\
0,708\end{array}$ & $\begin{array}{l}1,071 \\
1,049 \\
0,999\end{array}$ & $\begin{array}{l}G \times T \\
G \times R \\
R \times T\end{array}$ & $\begin{array}{l}\text { Sim } \\
\text { Não } \\
\text { Sim }\end{array}$ & $0,0371^{*}$ \\
\hline $\begin{array}{l}S / T \text { G M } \\
S / T R M \\
S / T \text { T M }\end{array}$ & $\begin{array}{l}26 \\
26 \\
26\end{array}$ & $\begin{array}{l}3,68 \\
4,13 \\
3,67\end{array}$ & $\begin{array}{l}1,360 \\
1,413 \\
1,382\end{array}$ & $\begin{array}{l}2,458 \\
2,320 \\
2,405\end{array}$ & $\begin{array}{l}G \times T \\
G \times R \\
R \times T\end{array}$ & $\begin{array}{l}\text { Não } \\
\text { Não } \\
\text { Não }\end{array}$ & 0,6006 \\
\hline $\begin{array}{l}\mathrm{l} / \mathrm{T} \mathrm{G} M \\
\mathrm{l} / \mathrm{T} \mathrm{R} M \\
\mathrm{l} / \mathrm{T} \mathrm{RO}\end{array}$ & $\begin{array}{l}26 \\
26 \\
26\end{array}$ & $\begin{array}{l}3,61 \\
3,86 \\
3,61\end{array}$ & $\begin{array}{l}1,397 \\
1,295 \\
1,463\end{array}$ & $\begin{array}{l}2,392 \\
2,337 \\
2,380\end{array}$ & $\begin{array}{l}G \times T \\
G \times R \\
R \times T\end{array}$ & $\begin{array}{l}\text { Não } \\
\text { Não } \\
\text { Não }\end{array}$ & 0,7654 \\
\hline $\begin{array}{l}S / N G M \\
S / N R M \\
S / N T M\end{array}$ & $\begin{array}{l}26 \\
26 \\
26\end{array}$ & $\begin{array}{l}2,95 \\
2,84 \\
3,12\end{array}$ & $\begin{array}{l}1,351 \\
1,285 \\
1,334\end{array}$ & $\begin{array}{l}2,146 \\
2,079 \\
2,051\end{array}$ & $\begin{array}{l}G \times T \\
G \times R \\
R \times T\end{array}$ & $\begin{array}{l}\text { Não } \\
\text { Não } \\
\text { Não }\end{array}$ & 0.1216 \\
\hline $\begin{array}{l}\mathrm{l} / \mathrm{N} G \mathrm{M} \\
\mathrm{l} / \mathrm{N} \mathrm{R} \mathrm{M} \\
\mathrm{I} / \mathrm{N} \mathrm{T} \mathrm{M}\end{array}$ & $\begin{array}{l}26 \\
26 \\
26\end{array}$ & $\begin{array}{l}3,07 \\
2,81 \\
2,80\end{array}$ & $\begin{array}{l}1,157 \\
1,028 \\
1,117\end{array}$ & $\begin{array}{l}2,105 \\
2,058 \\
1,990\end{array}$ & $\begin{array}{l}G \times T \\
G \times R \\
R \times T\end{array}$ & $\begin{array}{l}\text { Não } \\
\text { Não } \\
\text { Não }\end{array}$ & 0,7599 \\
\hline $\begin{array}{l}\text { T/N G M } \\
\text { T/N R M } \\
\text { T/N T M }\end{array}$ & $\begin{array}{l}26 \\
26 \\
26\end{array}$ & $\begin{array}{l}2,91 \\
2,19 \\
2,46\end{array}$ & $\begin{array}{l}0,565 \\
0,569 \\
0,601\end{array}$ & $\begin{array}{l}0,924 \\
0,912 \\
0,945\end{array}$ & $\begin{array}{l}G \times T \\
G \times R \\
R \times T\end{array}$ & $\begin{array}{l}\text { Não } \\
\text { Não } \\
\text { Não }\end{array}$ & 0,1464 \\
\hline
\end{tabular}

Para dar credibilidade ao aparelho, o conhecimento da reprodutibilidade intra e inter-observador é muito importante.

Sendo $\alpha=5 \%$ e considerando a média das imagens fornecida pelo programa do equipamento, não houve diferença estatisticamente significante entre as medidas de reprodutibilidade em nenhum parâmetro isolado analisado. Quando se analisou as relações entre os parâmetros isolados, apenas a relação $\mathrm{S} / \mathrm{I}$ mostrou diferença estatisticamente significante. Tal fato não foi observado quando se utilizou a melhor imagem escolhida por cada examinador, havendo diferenças estatisticamente significantes quanto aos parâmetros isolados inferior e temporal e quanto às relações $\mathrm{S} / \mathrm{T}, \mathrm{I} / \mathrm{T}, \mathrm{T} / \mathrm{N}$ e $\mathrm{S} / \mathrm{I}$.
Considerando $\alpha=1 \%$ para a significância estatística os dados obtidos com a melhor imagem tem uma reprodutibilidade ótima em todos os parâmetros exceto na relação $\mathrm{T} / \mathrm{N}$.

Assim os resultados deste estudo indicam que o exame se torna muito mais fidedigno se o examinador tomar três medidas de boa qualidade de cada olho e executar o cálculo da média.

Com as comparações múltiplas realizadas pelo método de Dunn não é possível encontrar um padrão de diferença entre um examinador e outro, deixando portanto de caracterizar tendenciosidade e sugerindo que o exame pode ser executado por qualquer pessoa, independentemente do grau de conhecimento em glaucoma. 


\begin{tabular}{|c|c|c|c|c|c|}
\hline Variável & $\mathbf{N}$ & Min & Max & Mediana & $\mathbf{P}$ \\
\hline $\begin{array}{l}\text { SG } 1 \\
\text { SG } 2 \\
\text { SG } 3\end{array}$ & $\begin{array}{l}26 \\
26 \\
26\end{array}$ & $\begin{array}{l}79,6 \\
83,8 \\
81,4\end{array}$ & $\begin{array}{l}149,8 \\
152,3 \\
160,1\end{array}$ & $\begin{array}{l}109,8 \\
113,6 \\
108,3\end{array}$ & 0,4815 \\
\hline $\begin{array}{l}\text { IG } 1 \\
\text { IG } 2 \\
\text { IG } 3\end{array}$ & $\begin{array}{l}26 \\
26 \\
26\end{array}$ & $\begin{array}{l}66,5 \\
69,4 \\
72,4\end{array}$ & $\begin{array}{l}137,9 \\
154,3 \\
149,2\end{array}$ & $\begin{array}{l}104,6 \\
104,6 \\
105,1\end{array}$ & 0,8910 \\
\hline $\begin{array}{l}\text { TG } 1 \\
\text { TG } 2 \\
\text { TG } 3\end{array}$ & $\begin{array}{l}26 \\
26 \\
26\end{array}$ & $\begin{array}{l}31,9 \\
26,6 \\
29,4\end{array}$ & $\begin{array}{l}90,2 \\
97,5 \\
93,5\end{array}$ & $\begin{array}{l}48,5 \\
49,2 \\
50,9\end{array}$ & 0,3035 \\
\hline $\begin{array}{l}\text { NG } 1 \\
\text { NG } 2 \\
\text { NG } 3\end{array}$ & $\begin{array}{l}26 \\
26 \\
26\end{array}$ & $\begin{array}{l}35,1 \\
32,5 \\
32,0\end{array}$ & $\begin{array}{l}87,2 \\
81,7 \\
78,4\end{array}$ & $\begin{array}{l}49,8 \\
51,6 \\
51,2\end{array}$ & 0,8910 \\
\hline $\begin{array}{l}\text { SR } 1 \\
\text { SR } 2 \\
\text { SR } 3\end{array}$ & $\begin{array}{l}26 \\
26 \\
26\end{array}$ & $\begin{array}{l}77,2 \\
74,8 \\
78,6\end{array}$ & $\begin{array}{l}150,7 \\
148,6 \\
155,0\end{array}$ & $\begin{array}{l}113,1 \\
112,2 \\
108,0\end{array}$ & 0,8910 \\
\hline $\begin{array}{l}\text { IR } 1 \\
\text { IR } 2 \\
\text { IR } 3\end{array}$ & $\begin{array}{l}26 \\
26 \\
26\end{array}$ & $\begin{array}{l}65,8 \\
63,4 \\
63,1\end{array}$ & $\begin{array}{l}148,2 \\
147,3 \\
144,6\end{array}$ & $\begin{array}{l}104,0 \\
107,9 \\
101,2\end{array}$ & 0,2410 \\
\hline $\begin{array}{l}\text { TR } 1 \\
\text { TR } 2 \\
\text { TR } 3\end{array}$ & $\begin{array}{l}26 \\
26 \\
26\end{array}$ & $\begin{array}{l}31,5 \\
25,7 \\
29,6\end{array}$ & $\begin{array}{l}96,4 \\
92,8 \\
95,4\end{array}$ & $\begin{array}{l}55,9 \\
50,5 \\
53,8\end{array}$ & 0,8910 \\
\hline $\begin{array}{l}\text { NR } 1 \\
\text { NR } 2 \\
\text { NR } 3\end{array}$ & $\begin{array}{l}26 \\
26 \\
26\end{array}$ & $\begin{array}{l}34,4 \\
36,6 \\
37,3\end{array}$ & $\begin{array}{l}76,7 \\
80,7 \\
84,9\end{array}$ & $\begin{array}{l}49,6 \\
52,5 \\
47,6\end{array}$ & 0,3035 \\
\hline $\begin{array}{l}\text { ST } 1 \\
\text { ST } 2 \\
\text { ST } 3\end{array}$ & $\begin{array}{l}26 \\
26 \\
26\end{array}$ & $\begin{array}{l}75,1 \\
73,4 \\
62,7\end{array}$ & $\begin{array}{l}151,6 \\
148,0 \\
152,7\end{array}$ & $\begin{array}{l}110,1 \\
108,4 \\
111,0\end{array}$ & 0,7640 \\
\hline $\begin{array}{l}\text { IT } 1 \\
\text { IT } 2 \\
\text { IT } 3\end{array}$ & $\begin{array}{l}26 \\
26 \\
26\end{array}$ & $\begin{array}{l}67,7 \\
73,0 \\
65,5\end{array}$ & $\begin{array}{l}150,8 \\
135,6 \\
148,4\end{array}$ & $\begin{array}{l}108,1 \\
105,2 \\
108,3\end{array}$ & 0,7074 \\
\hline $\begin{array}{l}\text { TT } 1 \\
\text { TT } 2 \\
\text { TT } 3\end{array}$ & $\begin{array}{l}26 \\
26 \\
26\end{array}$ & $\begin{array}{l}32,5 \\
30,5 \\
31,6\end{array}$ & $\begin{array}{l}86,0 \\
85,0 \\
92,8\end{array}$ & $\begin{array}{l}48,0 \\
46,1 \\
48,2\end{array}$ & 0,2231 \\
\hline $\begin{array}{l}\text { NT } 1 \\
\text { NT } 2 \\
\text { NT } 3\end{array}$ & $\begin{array}{l}26 \\
26 \\
26\end{array}$ & $\begin{array}{l}37,2 \\
36,8 \\
38,1\end{array}$ & $\begin{array}{l}77,5 \\
71,7 \\
82.9\end{array}$ & $\begin{array}{l}54,6 \\
54,0 \\
54,7\end{array}$ & 0.6303 \\
\hline
\end{tabular}

Há portanto uma excelente reprodutibilidade intra-observador entre os resultados dos exames realizados pelo $\mathrm{GD}_{\mathrm{X}}{ }^{\mathrm{MR}}$ em todas as regiões analisadas, e uma boa reprodutibilidade inter-observador quando os cálculos são realizados com a média das imagens.

\section{SUMMARY}

Purpose: To analyze the intra- and inter-observer reproducibility of retinal fiber layer measurements.

Methods: Twenty-six normal eyes underwent retinal nerve fiber layer thickness measurements with the GDx scanning system. Three images of each eye were taken by three different observers. For each case, each observer was asked to choose the best image and also to use the mean of the three images for each case. Comparisons were made using the best image and also the mean image provided by the software. The mean thickness values in each quadrant and their ratios were compared using Friedman rank analysis of variance.

Results: The comparison of results obtained from the three images taken by the same observer (intraobserver reprodutibility) did not show any statistically significant difference. The analysis of the data by the $R$ (resident), $T$ (technician) and $G$ (ophthalmologist specialized in glaucoma) observers revealed respective-ly: $p R(S=0.89 ; I=0.24 ; T=0.89 ; N$ $=0.30), p T(S=0.76 ; I=0.70 ; T=0.22 ; N=0.63)$, and $p G($ $S=0.48 ; I=0.89 ; T=0.30 ; N=0.89$ ). Regarding the isolated values (superior, inferior, temporal and nasal), the analysis of the results considering the values obtained from the mean image did not reveal a statistically significant difference between the three investigators. But when relationships were considered a statistically significant difference was observed regarding the superior/inferior ratio ( $p S / I=0.037)$. Statistically significant differences were detected when considering the best image. Inferior and temporal mean thickness values were statistically different between observers ( $p=0.04$ and 0.003 respectively). Also, the superior/temporal $(S / T)$, inferior/temporal (I/T), temporal/nasal (T/N) and superior/inferior (S/I) ratios showed statistically significant differences between observers ( $p S / T$ $=0.02 ; p I / T=0.03 ; p T / N=0.007 ; p S / I=0.037)$.

Conclusion: The results showed good reproducibility of the GDx Scanning System results using the mean image provided by the equipment software.

Keywords: GDx; Nerve fiber analyzer; Nerve fiber layer.

\section{REFERÊNCIAS BIBLIOGRAFICAS}

1. Shields MB. Glaucoma Screening. In: Shields MB Textbook of Glaucoma. Baltimore, Williams \& Wilkins, 4th edition 1998:137 (cap 7).

2. Jonas JB, Schiro D. Localised wedge shaped defects of the retinal nerve fiber layer in glaucoma. Br J Ophthalmol 1994;78:285-90.

3. Quigley HA, Dunkelberger GR, Green WR. Retinal ganglion cell atrophy correlated with automated perimetry in human eyes with glaucoma. Am J Ophthalmol 1989;107:453-64.

4. Sommer A, D'Anna SA, Kues HA, George T. High resolution photography of the retinal nerve fiber layer. Am J Ophthalmol 1983;96:535-9.

5. Airaksinen PJ, Nieminen H. Retinal nerve fiber layer photography in glaucoma. Ophthalmology 1985;92:877-9.

6. Dreher AW, Reiter K. Retinal laser ellipsometry: a new method for measuring the retinal nerve fiber layer thickness distribution? Clin Vis Sci 1992;7:481-8.

7. Weinreb RN, Dreher AW, Coleman A, Quigley HA, Shaw B, Reiter K. Histopathologic validation of Fourier-ellipsometry measurements of retinal nerve fiber layer thickness. Arch Ophthalmol 1990;108:557-60.

8. Tjon-Fo-Sang MJ, Lemij HG. The sensitivity and Specificity of nerve fiber layer measurements in glaucoma as determined with Scannig Laser Polarimetry. Am J Ophthalmol 1997;123:62-9.

9. Suzanna Jr R, Takahashi WY, Nakamura NKF. Sensibilidade e especificidade da camada de fibras nervosas examinadas através da polarimetria a laser. Rev Brasil Oftalmol 1998;57(1):17-22. 
10. Weinreb RN, Shakiba S, Zangwill L. Scanning laser polarimetry to measure the nerve fiber layer of normal and glaucomatous eyes. Am J Ophthalmol 1995;119:627-36.

11. Chi Q, Tomita G, Inazumi K, Hayakawa T, Tadayoshi I, Kitazawa Y. Evaluation of the effect of aging on the retinal nerve fiber layer thickness using scanning laser polarimetry. J Glaucoma 1995;4:406-13.

12. Zangwill L, Berry CA, Garden VS, Weinreb RN. Reprodutibility of retardation measurements with the nerve fiber analizer II. J Glaucoma 1997;6:384-9.
13. Tjon-Fo-Sang MJ, Strik van R, Vries J, Lemij HG. Improved Reprodutibility of measurement with the nerve fiber analizer. J Glaucoma 1997;6:203-11.

14. Holló G, Nagymihály A, Vargha P. Scanning Laser Polarimetry in cornel haze after Excimer Laser refractive surgery. J Glaucoma 1997;6:359-62.

15. Swanson WH, Lynn JR, Fellman RL, Starita RJ, Schumann SP, Nusinowitz S. Interoperator variability in images obtained by laser polarimetry of the nerve fiber layer. J Glaucoma 1995;4:414-8.

\section{SIMPÓSIO MOACYR ÁLVARO VÍTREO - RETINA - ÚVEA - AIDS -TRAUMA - LASER "PRESENTE E FUTURO" UNIFESP - ESCOLA PAULISTA DE MEDICINA 24 a 26 de Fevereiro de 2000}

APO I 0: Sociedade Brasileira de Retina e Vïtreo, Uveíte, Trauma, Laser e Cirurgia Oftalmológica

Estamos orgulhosos em anunciar a realização do primeiro evento anual mais importante do Departamento de Oftalmologia da UNIFESP / EPM, a ser realizado no próximo século, em 24 a 26 de Fevereiro do ano 2000, com apoio das Sociedades Brasileiras de Retina e Vítreo, Uveíte, Trauma, Laser e Cirurgia Oftalmológica.

Haverá participação de vários convidados estrangeiros e nacionais. Serão promovidos cursos teóricos, práticos, básicos e avançados com ênfase na apresentação de um festival de casos clínicos e cirúrgicos, anátomo-patológicos, vídeos, temas livres e posters com ampla discussão. Serão apresentadas cirurgias com interação dinâmica em tempo real. Haverá concursos de imagem e fotografia e prêmios para os melhores trabalhos.

Prepare-se para desfrutar da fascinação e dos avanços em segmento posterior e reserve os dias 24 a 26 de Fevereiro do ano 2000 em sua agenda para 0 III SIMAsp.

\section{COMISSÃO ORGANIZADORA \\ Dr. Michel Eid Farah \\ Dr. Rubens Belfort Jr. \\ Dr. José Carlos Reys \\ Dra. Ana Luisa Höfling de Lima \\ Dra. Nilva Simeren B. Moraes}

I N F O R M A Ç ÕE S: JDE Comunicação e Eventos

Al. Santos, 705 Cj. 56 - CEP 01419-001 - São Paulo - SP

Tel: (011) 289-4301 - 251-5273

Fax: (011) 288-8157 - E-mail: jdecomev@vol.com.br 